\title{
Disaster Management Based on IoT Enable Monitoring Technique
}

\author{
${ }^{1}$ Madhura S, ${ }^{2}$ Disha D, ${ }^{3}$ Deepthi G, ${ }^{4}$ Chihnitha B \\ $1,2,3,4 \mathrm{RV}$ Institute of Technology and Management, Bangalore, India \\ 1madhu4tuip@gmail.com \\ ${ }^{2}$ dishad is19.rvitm@rvei.edu.in \\ 3 deepthig_ec19.rvitm@rvei.edu.in \\ ${ }^{4}$ chihnithab ec19.rvitm@rvei.edu.in
}

\begin{abstract}
The disasters can be either natural or man-made. Control and management of disaster of any kind is possible in effective and robust way by the implementation of IoT in the system. The objective of implementing IoT into the disaster management system is the quick and effective recovery from the disaster. The various methods that can be deployed after the disaster is outlined through utilization of IoT. This paper gives an insight on the various methods that can be effectively used after the disaster using IoT. The existing techniques are very well monitored and has the ability to react to the situation as per needs, this paper significantly provides the contribution in analyzing these techniques for appropriate disaster management development block.
\end{abstract}

Keywords- Geographical Information System, Security Monitoring, Nerve Net, Drone, Disaster Management

\section{INTRODUCTION}

Internet of things is an interconnection of devices with embedded communication and sensing possibilities. IoT gives the opportunity for different devices to communicate not only within the silos but across varied networking types and creates a more connected world. It plays a key role for realizing the dynamic workflow adaptations and has potential to communicate the updated instantaneous information [1]. It is an important driver for data-driven optimization and automation, customer-facing innovation, digital transformation, revenue streams, Research and Development and innovative applications, and business models across every sector [2]. The data can be analyzed, gathered, communicated and aggregated through uniquely identifiable IP addresses which are directly connected to the physical end points of IOT. It 
Journal of Trends in Computer Science and Smart technology (TCSST) (2021)

Vol.03/ No. 02

Pages: $70-80$

https://www.irojournals.com/tcsst/

DOI: https://doi.org/10.36548/jtcsst.2021.2.001

helps in identification of life-threatening hazards and immediately gives warning which helps in evacuation of people from the affected areas, provide effective support through relief workers, sending ambulance drones in disaster areas.

\section{Disaster MANAGEMENT}

The man being the most advanced species on the planet earth has control over the most things happening around him [3]. However, nature and its wild forces can't be controlled by anyone. We can however predict the disasters in advance and evacuate people and prevent the loss of lives.

Disasters can be classified into severe, moderate and mild. Depending on the type, severity of the calamity and the region affected different drones are used [4]. For example: The response for a high magnitude earthquake and slow onset calamity like floods cannot be the same., hence devices are designed for a specific purpose.

IOT and WSN sensors help in detecting and forecasting disaster like floods; many several sensors also have audio and visual devices like sirens and light which help in warning the localities. These devices enable different features to monitor the environment and update these data to the Tier $2 /$ fog computing

It's necessary to have our town infrastructure planned and prepared for the disasters.

There are many structures such as high-rise buildings or bridges that have built-in sensors whose conditions are continuously monitored through geographic information system (GIS) and satellite communication. From these sources of data, we can improve early warning systems, use social media to create awareness about imminent earthquakes ad so on. An early warning can be given by the different type of shock waves a tremor emits [6]. As well as Ground movement acceleration sensors can trigger early warnings of earthquake. In case of forest fires sensors attached to trees record parameters which change during these situations like $\mathrm{CO}$ and $\mathrm{CO} 2$ level, humidity, temperature. If theses parameters reach critical levels then it notifies the fire service station. These sensors provide GPS coordinates which helps the rescue team and firefighters to localize the fire [7]. The combination between IOT and new advanced technologies like Wireless Sensor Networks (WSN), is one of the most promising solution to help deal with the problem of natural disasters. 
Journal of Trends in Computer Science and Smart technology (TCSST) (2021)

Vol.03/ No. 02

Pages: $70-80$

https://www.irojournals.com/tcsst/

DOI: https://doi.org/10.36548/jtcsst.2021.2.001

\section{DRONES AND IOT}

IoT plays an important role in both pre, post and during the disaster. IoT that finds its application in drones helps disaster management significantly. Drones find their reach in all the areas that are inaccessible for a man and find a vast application in various fields: Military, Emergency Services, Disaster Management, Security Monitoring and other commercial applications. Processing of the accumulated information by IoT devices is required to identify the severely affected areas and to support people where human intervention is risky, impossible, hazardous, and expensive [8]. A drone with IoT devices can be used to gather data from disaster area and its surroundings. The camera drone in the air can take images of the scenario and send them to rescue team so that they can respond faster during critical situations. A drone usually lifts up a flying platform by incorporating some communication technologies such as WiMAX, or LTE, ad hoc, Wi-Fi, equipment which provide an efficient communication service, where usually a disaster generally tends to destroys all the communication and electric supply. The camera drone in the air can take images of the situation and send them to the rescue team so that they can respond faster during critical situations. Ambulance drones are quicker than any ground vehicle emergency vehicle in exceptionally thick urban communities. Drones are one of the most encouraging innovations to improve firefighting reaction and direct the salvage tasks more secure, quicker and effective when contrasted with conventional monitored held activities.

\section{FIRES}

Fires are disastrous and like any other calamity causes a huge loss of lives and property. High rise buildings under fire accidents are dangerous affecting thousands of people at once. New Urban Infrastructure equipped with sprinklers, fire extinguishers, proper fire exits are necessary and now is mandated by the government bodies all around the world.

Sangmin Park et. al. propose a 3-step model to deal with fire disasters.

\section{Intelligent Service:}

It is important to supply data that envisions Augmented reality based solutions that classifies a hazardous environment and provides a safe evacuation route at the site of fire. This portrays the infrastructural plan in both physical and virtual realm of buildings [9]. 
Journal of Trends in Computer Science and Smart technology (TCSST) (2021)

Vol.03/ No. 02

Pages: $70-80$

https://www.irojournals.com/tesst/

DOI: https://doi.org/10.36548/jtcsst.2021.2.001

\section{Safety Standard:}

It is vital to have a safe operating procedure by providing standard guidelines to the residents and rescuers. This helps scale back on the casualties caused by poor choice of evacuation routes due to lack of visibility during the fires.

\section{Internet of Things Based Real-Time Remote System:}

IoT-based systems is predicted to be in connection with the fire department. The remote system will be equipped with rapid response through providing the locals with real-time results.

- Smart sensor-based real time fire detection: Gathering and monitoring of smart sensors providing real time data as mentioned in Table [1] and sending out the report of the state to a server as soon as there is a detection of fire [10].

- Transmit the sensed information: Smart Zigbee Gateway is used to sense and transmit real time information to the servers.

- AR-based fire occurrence monitoring: Information transmitted to the server is processed and analyzed. This location information and the severity is displayed on the User Interface [UI] based on AR like smart tablets, phones etc. AR based User Interface helps in timely rescue by notifying the concerned authorities about the guidelines to be followed for evacuation.

Agoston Restas suggests the use of drones to detect hotspots quickly and give information to the fire department. The drones are very effective as a large area can be monitored and the information can be sent real-time [11]. Drones equipped with high definition and smart sensors help in giving a first-hand information about the fire like the severity of fire, affected areas, direction of wind, smoke emission, speed of the fire spreading and so on [22-25].

The use of unmanned aerial vehicles is accommodating where the disaster is moderate and is spread across a large area. For large disasters like forest fires, they act as a supporting tool and 
Journal of Trends in Computer Science and Smart technology (TCSST) (2021)

Vol.03/ No. 02

Pages: $70-80$

https://www.irojournals.com/tcsst/

DOI: https://doi.org/10.36548/jtcsst.2021.2.001

TABLE I.

SMART IOT BASED REAL-TIME REMOTE SYSTEM

Description

Cognitive Sensor

Extinction

Server

Sensor

Prevention of fire in

buildings

Initial Ignition:

Smart integrated gas

sensor for smoke

detection.

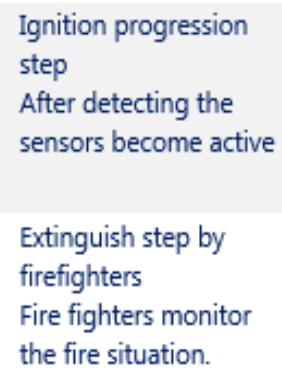

Electric leak detector
Smart gas leakage
sensor
Multi gas detector

Multi gas detector
Flame detector
Smoke detector
CCTV
Occupant Sensor
Temperature Sensor
Flame detector
Smoke detector
CCTV
Occupant Sensor
Temperature and
Occupant Sensor

$-$

Server

Smart gas Smart Zigbee

leakage gateway

sensor Server

Electric

leak

detector

Smart Zigbee

gateway

Server

Multi gas Smart Zigbee

detector gateway

CCTV Server

Flame

detector

Smoke

detector

Smart Zigbee

gateway

Server

Smart Zigbee

gateway

Server

\section{FLOODS}

India being tropical in south and temperate in the Himalayan regions, receives abundant rainfall. India has been a witness to floods almost every year may it be through heavy rains causing dams to flood or like the terrifying flash floods of Kedarnath 2013. The more recent Hyderabad floods of 2020, disrupted the lives of people overnight. In the difficult times of Novel Corona Virus people were stranded as their homes were flooded.

i. What could IoT have done differently?

ISSN: 2582-4104

Submitted:25.05.2021 
Journal of Trends in Computer Science and Smart technology (TCSST) (2021)

Vol.03/ No. 02

Pages: $70-80$

https://www.irojournals.com/tcsst/

DOI: https://doi.org/10.36548/jtcsst.2021.2.001

Ghapar, A et. al. has proposed a 3-step solution -

- Perception layer

- Network Layer

- Application layer.

Perception Layer: This is the layer where all the data arises and it is the most important layer of Internet of Things [12]. The prime objective of this step is to collect information from the surrounding location using sensors and actuators. This layer after detecting and collecting data processes it and passes it on to the next layer.

Network Layer: This layer is responsible for routing the data and it transmits this data to the subsequent stage of upper layer. The web, wireless and mobile communication network are used to transmit this information.

Application Layer: The function of application layer is to collect, assimilate and store the information. It provides good UI and caters to the precise need of the user.

Partha Pratim et. al. suggests a visual flood monitoring system. In this system, CCTV cameras are used where real time images of the river are captured and sent to machines that are remotely located. Here, ML algorithms helps in deciding the status of the flood and level of water in the river [13]. When the threshold level is crossed, a push notification and an alarm is initiated as a warning. To begin with, the images are taken via the CCTV cameras that are enabled with remote IP. Succeeding this, the pictures are added with a real-time feed and virtual pointers. This is followed by a visual sensing which is occurrence based. That's recorded as floor risk and valuation of the water-level. To conclude, the recorded levels of fluctuation is continually sent to the system [14]. This event-based activating process is achieved in two steps - Initially, all the noises are processed using an image processor and then marked for subsequent feeding; Next, the supply of water is searched by an algorithm by the proposed method.

\section{ii. FLOOD DETECTION NETWORK:}

It is an IoT-enabled communication which is used for open crowd sensing that is linked further to flood sensing nodes round the world. Currently, this network is executed in Oxford floodplain. The present designs of the node consist of ultrasonic range finder, Raspberry Pi, and router. There is a node usually distributed under the river bridge which measures the 
Journal of Trends in Computer Science and Smart technology (TCSST) (2021)

Vol.03/ No. 02

Pages: $70-80$

https://www.irojournals.com/tcsst/

DOI: https://doi.org/10.36548/jtcsst.2021.2.001

altitude of water at every minute period. The information is then sent to the mapped service of distant cloud which is to be found at the website http://map.ood.network it is used for real time monitoring of existing flood situation. If the altitude of water exceeds the safety level, the map changes its color to yellow or red for illustration. At the same instant, the notification is also sent to local control center and to the people related over the web [16].

\section{iii. FLOATING SENSOR NETWORKS:}

There is a latest progression in UC Berkley which has opened a substitute paradigm using the floating product of collecting information about flood circumstances of a river. The value and portable efficient floating object has acceleration sensor and (GPS) Global Positioning System sensor. Slow change or rapid rise in water altitude is observed by floating object and instantaneously sent to the local people through web and alerts.

\section{iv. EARTHQUAKE:}

Earthquakes destroy lives and property and cause permanent damage. Researchers are constantly working to improve the IoT-based system which helps to update the distantly located victims before the incident takes place. Partha Pratim recommended the following model 'Nerve Net\&\#39' which is implemented at Onagawa, Japan, which is one of the main advancement on IoT integration with earthquake monitoring. It was created on the basic concept of bypass network which is already verified to be a disaster resilient [17]. This network is internationally circulated over a wide range of a locality where distant and local interactions happen by involving optical Ethernet, WI-FI, Unmanned Aerial Vehicle (UAV) and satellite.However, any earthquake related systems effectiveness depends on its previous exchange of information. To cope with the issue, an Iot built warning system is intended. For ZigBee communication, the planned classification is made where the PIC microcontroller does all the data processing work. The information is retrieved by a variety of accelerometers from multiple locations on the grounds and gathered at the server end. However, if the measurement obtained is above the magnitude, the device alerts the people in the crowd about the significant hazard.

Rustam Pirmagomedov et. al. indicates that the use of living creatures as earthquake sensors could complement the prevailing tectonic activity prediction developments and thus the forthcoming advancements. It is important to design the device structure in a modular manner 
Journal of Trends in Computer Science and Smart technology (TCSST) (2021)

Vol.03/ No. 02

Pages: $70-80$

https://www.irojournals.com/tesst/

DOI: https://doi.org/10.36548/jtcsst.2021.2.001

to realize the objective. Using standard interfaces, such a design will allow easy integration of various elements [18].

Motor sensory is amongst the key animal response to an external stimuli. It is important to record movements within the time frame in order to observe body movements [15]. This can be achieved both with the guidance of nationwide navigation (GPS) and with the help of automated inertial guidance applications. The use of acceleration sensors and gyroscope is assisted by such systems. It is possible to measure (in a reasonable error margin) the distance covered by the object from the information obtained and even identify individual movements. For the collection and review of information on the motor movements of animals residing clo se to humans (like puppies and cats), as well as animals such as horses, cows or sheep, th e occurrence of a mechanism for observing animal activity

with the aid of wearable sensing devices.

\section{CONCLUSION}

IoT based disasster management is a multistep process that requires user interaction between each step amd quick action from the early warning. The after effects of the disaster can be minmized by formation of appropriate public policies and better stratergy. The main objective of this paper is to provide different types of natural and man made disaster that can be solved and better management by various methods proposed by the different researchers has been affectively presented.

\section{REFERENCES}

[1] Sangmin Park, Soung Hoan Park, Lee Won Park, Sanguk Park, Sanghoon Lee, Tacklim Lee, Sang Hyeon Lee, Hyeonwoo Jang, Seung Min Kim, Hangbae Chang and Sehyun Park,"Design and Implementation of a Smart IoT Based Building and Town Disaster Management System in Smart City Infrastructure"

[2] Agoston Restas, “ Drone Applications for Supporting Disaster Management”, World Journal of Engineering and Technology, 2015, 3, 316-321 Published Online October 2015 in SciRes. http://www.scirp.org/journal/wjet, http://dx.doi.org/10.4236/wjet.2015.33C047

[3] Partha Pratim Ray, Mithun Mukherjeeand Lei Shu, “ Internet of Things for Disaster Management: State-of-the-Art and Prospects", IEEE Acces, DOI: 10.1109/ACCESS.2017.2752174 
Journal of Trends in Computer Science and Smart technology (TCSST) (2021)

Vol.03/ No. 02

Pages: $70-80$

https://www.irojournals.com/tcsst/

DOI: https://doi.org/10.36548/jtcsst.2021.2.001

[4] S. H. Alsamhi, O. Ma, M. S. Ansari and F. A. Almalki, "Survey on Collaborative Smart Drones and Internet of Things for Improving Smartness of Smart Cities," in IEEE Access, vol. 7, pp. 128125-128152, 2019, doi: 10.1109/ACCESS.2019.2934998.

[5] Sinha, A., Kumar, P., Rana, N.P. et al. Impact of internet of things (IoT) in disaster management: a task-technology fit perspective. Ann Oper Res 283, 759-794 (2019). https://doi.org/10.1007/s10479-017-2658-1

[6] Ghapar, A. A., S. Yussof and Asmidar Abu Bakar. "Internet of Things (IoT) Architecture for Flood Data Management." (2018). International Journal of Future Generation Communication and Networking Vol. 11, No. 1 (2018), pp.55-62 http://dx.doi.org/10.14257/ijfgcn.2018.11.1.06

[7] Rustam Pirmagomedov, Mikhail Blinnikov, Alexey Amelyanovich, Ruslan Glushakov, Svyatoslav Loskutov, Andrey Koucheryavy, Ruslan Kirichek, Ekaterina Bobrikova, "IoT Based Earthquake Prediction Technology”, Springer Professional.

[8] E. Vattapparamban, İ. Güvenç, A. İ. Yurekli, K. Akkaya and S. Uluağaç, "Drones for smart cities: Issues in cybersecurity, privacy, and public safety," 2016 International Wireless Communications and Mobile Computing Conference (IWCMC), Paphos, 2016, pp. 216221, doi: 10.1109/IWCMC.2016.7577060.

[9] H. N. Saha et al., "Disaster management using Internet of Things," 2017 8th Annual Industrial Automation and Electromechanical Engineering Conference (IEMECON), Bangkok, 2017, pp. 81-85, doi: 10.1109/IEMECON.2017.8079566.

[10] Gaire R. et al. (2020) Internet of Things (IoT) and Cloud Computing Enabled Disaster Management. In: Ranjan R., Mitra K., Prakash Jayaraman P., Wang L., Zomaya A.Y. (eds) Handbook of Integration of Cloud Computing, Cyber Physical Systems and Internet of Things. Scalable Computing and Communications. Springer, Cham. https://doi.org/10.1007/978-3-030-43795-4_12

[11] Zelenkauskaite, N. Bessis, S. Sotiriadis and E. Asimakopoulou, "Disaster Management and Profile Modelling of IoT Objects: Conceptual Parameters for Interlinked Objects in Relation to Social Network Analysis," 2012 Fourth International Conference on Intelligent Networking and Collaborative Systems, Bucharest, 2012, pp. 509-514, doi: 10.1109/iNCoS.2012.26. 
Journal of Trends in Computer Science and Smart technology (TCSST) (2021)

Vol.03/ No. 02

Pages: $70-80$

https://www.irojournals.com/tcsst/

DOI: https://doi.org/10.36548/jtcsst.2021.2.001

[12] Madhura S, Shubham Luharuka, Gaurav Anil Kulkarni, Pallothu Devi Shree, Ria Somani, "Technical Scrutiny of Block chain Technology Protocols and its Applications", IRO Journal on Sustainable Wireless Systems (2020), Vol.02/ No. 1 Pages: 13-22 http://irojournals.com/irosws/ DOI: https://doi.org/10.36548/jsws.2020.1.002

[13] S. R. Vijayalakshmi and S. Muruganand, "A survey of Internet of Things in fire detection and fire industries," 2017 International Conference on I-SMAC (IoT in Social, Mobile, Analytics and Cloud) (I-SMAC), Palladam, 2017, pp. 703-707, doi: 10.1109/ISMAC.2017.8058270.

[14] Adeel A. et al. (2019) A Survey on the Role of Wireless Sensor Networks and IoT in Disaster Management. In: Durrani T., Wang W., Forbes S. (eds) Geological Disaster Monitoring Based on Sensor Networks. Springer Natural Hazards. Springer, Singapore. https://doi.org/10.1007/978-981-13-0992-2_5

[15] P. Ghasemi and N. Karimian, "A Qualitative Study of Various Aspects of the Application of IoT in Disaster Management," 2020 6th International Conference on Web Research (ICWR), Tehran, Iran, 2020, pp. 77-83, doi: 10.1109/ICWR49608.2020.9122323.

[16] M. Kamruzzaman, N. I. Sarkar, J. Gutierrez and S. K. Ray, "A study of IoT-based postdisaster management," 2017 International Conference on Information Networking (ICOIN), Da Nang, 2017, pp. 406-410, doi: 10.1109/ICOIN.2017.7899468.

[17] Madhura S, “A Secure Protocol for Smart Meters using IoT Enabled Distribution Networks and Blockchain Security Mechanism", Journal of Ubiquitous Computing and Communication Technologies (UCCT) (2020) , Vol.02/ No. 01 Pages: 48-58 https://www.irojournals.com/jucct/ DOI: https://doi.org/10.36548/jucct.2020.1.006,

[18] ZAHRA, Syeda Ambreen; SHAFIQUE, Iqra; FARID, Tuba. Internet of Things (IoTs) For Disaster Management. Sukkur IBA Journal of Computing and Mathematical Sciences, [S.1.], v. 2, n. 1, p. 77-85, june 2018. ISSN 2522-3003.

[19] Chen, Joy Iong-Zong, and Jen-Ting Chang. "Route Choice Behaviour Modeling using IoT Integrated Artificial Intelligence." Journal of Artificial Intelligence 2, no. 04 (2020): 232237.

[20] Chen, Dr Joy Iong Zong, and Kong-Long Lai. "Internet of Things (IoT) Authentication and Access Control by Hybrid Deep Learning Method-A Study." Journal of Soft Computing Paradigm (JSCP) 2, no. 04 (2020): 236-245. 
Journal of Trends in Computer Science and Smart technology (TCSST) (2021)

Vol.03/ No. 02

Pages: $70-80$

https://www.irojournals.com/tcsst/

DOI: https://doi.org/10.36548/jtcsst.2021.2.001

[21] Chen, Joy Iong-Zong, and Lu-Tsou Yeh. "Greenhouse Protection Against Frost Conditions in Smart Farming using IoT Enabled Artificial Neural Networks." Journal of Electronics 2, no. 04 (2020): 228-232.

[22] Smys, S. "A Novel Multi-Tier Architecture Based Mobile Cloud Computing For Enhanced Energy Utilization." Journal of ISMAC 2, no. 01 (2020): 62-72.

[23] Chen, Joy Iong Zong. "Optimal Multipath Conveyance with Improved Survivability for WSN's In Challenging Location." Journal of ISMAC 2, no. 02 (2020): 73-82.

[24] Shakya, Subarna, and Lalitpur Nepal Pulchowk. "The Robust Routing Protocol with Authentication for Wireless Adhoc Networks." Journal of ISMAC 2, no. 02 (2020): 83-95.

[25] Bashar, Abul. "Sensor Cloud Based Architecture with Efficient Data Computation and Security Implantation for Internet of Things Application." Journal of ISMAC 2, no. 02 (2020): 96-105 